\title{
Metabolic reprogramming as a continuous changing behavior of tumor cells
}

\author{
Silvia Peppicelli ${ }^{1}$ Francesca Bianchini $^{1} \cdot$ Lido Calorini $^{1}$
}

Received: 17 April 2015 / Accepted: 3 July 2015 / Published online: 10 July 2015

(C) International Society of Oncology and BioMarkers (ISOBM) 2015

\begin{abstract}
Malignant cells resist microenvironment stress and migrate into surrounding tissues in order to divide with the need to adapt their metabolic program. These changes, often strengthened by the tremendous liaison between hypoxia, low glucose, and acidosis, are not yet completely understood. The aim of this perspective is to re-organize a possible comprehensive scenario useful to identify the metabolism occurring in various tumor cell subpopulations endowed with different capabilities.
\end{abstract}

Keywords Cancer metabolism · Acidic microenvironment . Hypoxia

\section{Metabolic reprogramming: a continuous changing behavior of tumor cells}

Most of the drugs used to target selectively and to kill advanced tumor cells have not been found useful to control disease progression; thus, new alternative therapeutic approaches are needed. Now, advances in analytical tools and progress in cancer genomics stimulate us to re-consider metabolism as a possible target for new therapeutic strategies. However, the metabolic program in tumor cells changes continuously to adapt to different microenvironment stressors; indeed, the metabolic plasticity of tumor cells is considered a hallmark of cancer [1]. It is known that malignant cells, in

Lido Calorini

lido.calorini@unifi.it

1 Department of Experimental and Clinical Biomedical Sciences, Section of Experimental Pathology and Oncology, Florence University, Viale G.Morgagni, 50, Florence 50134, Italy order to proliferate, resist microenvironmental changes and migrate into surrounding host tissues, need to adapt their metabolic profile switching back and forth from aerobic/anaerobic glycolysis to oxidative phosphorylation (OxPhos).

It is recognized that dividing tumor cells adopt "aerobic glycolysis", the so-called "Warburg effect"[2]. This means that tumor cells undergoing division, also with plenty of oxygen tension, prefer glycolysis, which combined with tricarboxylic acid (TCA) cycle activity ensures biomass formation, DNA synthesis and enough energy. At variance with the Warburg hypothesis, an intact mitochondrial activity is required, and tumor cells prevent mitochondrial disruption by the loss of TCA intermediates exited for anabolic purposes increasing this way glutamine uptake and lysis (anaplerosis) [3]. Considering Weinhouse's observation, that tumor cells consume oxygen at a rate similar or higher than their normal counterparts [4], it is possible that a mitochondrial uncoupling, in the absence of oxygen consumption defects, leads to "aerobic glycolysis" adoption [5]. The continual supply of glucose and other nutrients in circulating blood assures a high glycolytic flux able to yield a sufficient ATP production. However, glycolytic flux exceeds pyruvate dehydrogenase's (PDH) activity used for pyruvate entry into the TCA cycle, and pyruvate is converted by the lactate dehydrogenase A (LDH-A) to lactate and protons, which need to be secreted out of the cells to avoid cytoplasm acidity and stop of proliferation. In this way, extracellular $\mathrm{pH}$ starts to reduce [6].

At the same time, increasing the distance from supporting blood vessels, oxygen availability is reduced although it is not the primary fuel for the proliferating tumor cell subpopulation. Compared to glucose, oxygen has a greater diffusion coefficient, but a worse solubility causing a shorter diffusion distance and sudden $\mathrm{pO}_{2}$ reduction from vessels to the periphery (diffusion-limited ischemic hypoxia). Furthermore, cytokine production by either tumor cells or host inflammatory cells 
drives a slow systemic inflammation [7], which may contribute to the development of the so-called "anemic hypoxia" [8]. The following three causative mechanisms cooperate to anemia: a) a failure of bone marrow erythropoiesis to meet demand from tissues (hypoproliferative anemia), b) a defective iron reutilization due to intracellular iron sequestration promoted by hepcidin, an acute phase reactant protein (iron-deficiency anemia), and c) a shortened red cell survival induced by procoagulant proteins (microangiopathic hemolitic anemia) [9]. All of the above mentioned mechanisms, often associated with direct effects of cancer such as blood loss, bone marrow replacement, and side effects of tumor therapy, get worse tumor oxygenation already demanding by anemia of tumor-bearing patients. Thus, tumor tissues are invariably hypoxic with an average $\mathrm{pO}_{2}$ of $<10 \mathrm{mmHg}$ when $\mathrm{Hb}$ is $<13 \mathrm{~g} / \mathrm{dl}$ [10]. Evidence has indicated that recombinant erythropoietin can be used as a useful tool to ameliorate anemia in many cancer patients, and a recent paper by Murayama et al. (2014) shows how the level of Hb may help standard chemotherapy to suppress metastasis [11].
The ability of tumor cells to adapt themselves to a reduced oxygen tension relies on the hypoxia-inducible factor (HIF)$1 \alpha$ transcription factor, which is regulated at the protein level in an oxygen-sensitive manner. HIF- $1 \alpha$-related genes are critical in inducing reduction of cell proliferation combined with the development of a more aggressive and resistant phenotype particularly potentiated by an epithelial-to-mesenchymal transition (EMT) program $[12,13]$. HIF-1 $\alpha$-dependent anaerobic glycolysis supports energy requested by hypoxic tumor cells, and pyruvate dehydrogenase (PDH) kinase 1 activation suppresses PDH complex, limiting the entry of pyruvate into the TCA cycle. Thus, pyruvate is entirely redirected into lactate production by LDH-A, an activity also under HIF- $1 \alpha$ control. To avoid damage by acidity, lactate and protons are transported out of the cells by monocarboxylate transporter 4 , an additional target of HIF-1 $\alpha$ [13]. Protons can also be exported out of the cells by $\mathrm{Na}^{+} / \mathrm{H}^{+}$exchangers. Both aerobic and anaerobic glycolysis of dividing and hypoxic tumor cells, respectively, participate to establish a "reversed" $\mathrm{pH}$ gradient between intra and extracellular compartment of tumor cells, so

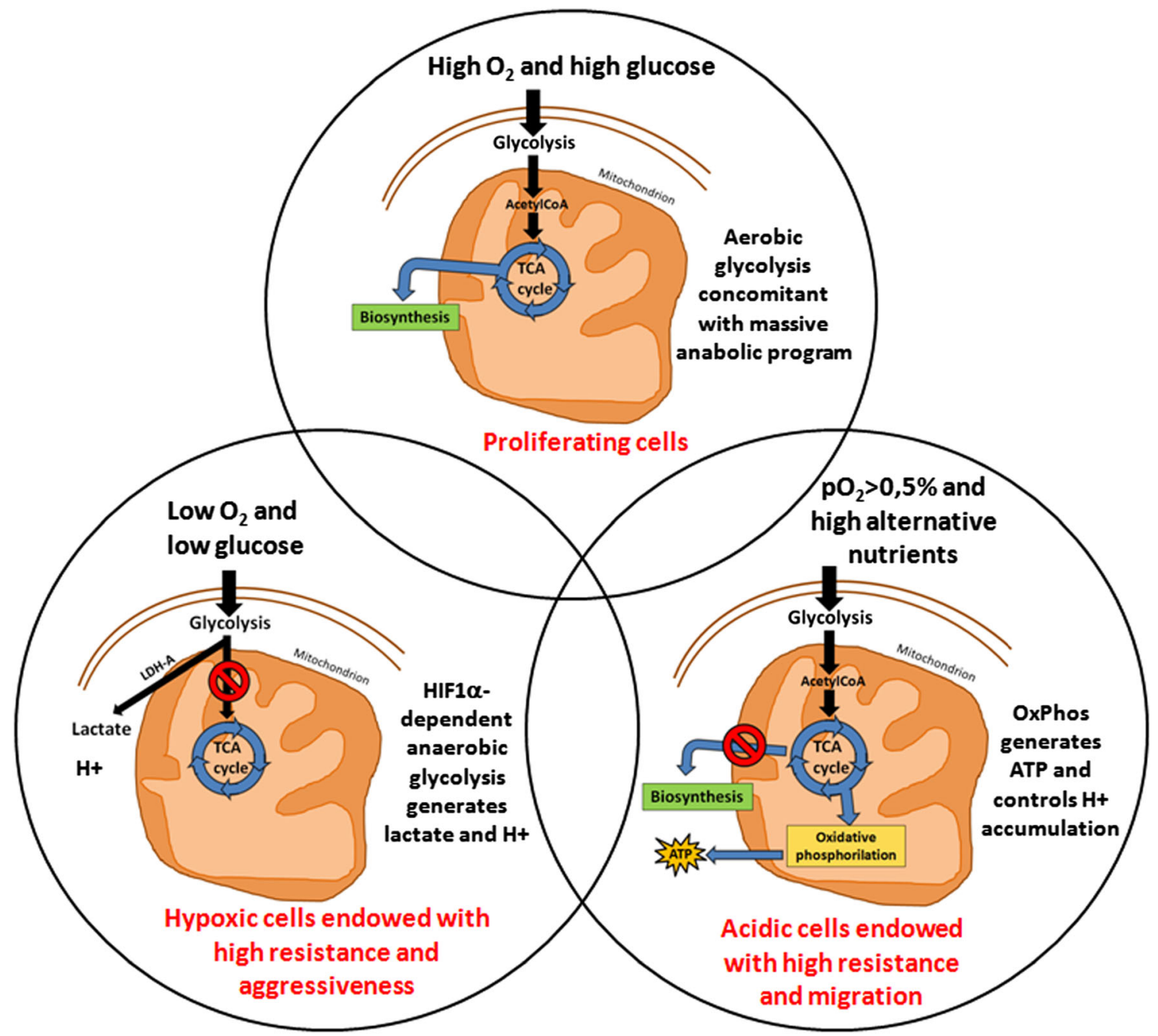

Fig. 1 "Triade" of major microenvironmental determinants affecting metabolism and biological properties of tumor cells 
adapted cancer cells produce significant acidification of the extracellular microenvironment, maintaining a normal or slightly alkaline internal $\mathrm{pH}$. We cannot exclude that a chaotic vasculature, low lymphatic drainage, and high interstitial pressure may contribute in lowering extracellular $\mathrm{pH}(\mathrm{pHe})$ [14]. Acidity generated by protons and lactate correlates with a poorer clinical prognosis and resistance to standard therapy; likewise, it stimulates EMT, invasiveness, and metastatic spread of tumor cells, reducing proliferation [15]. Low pHe may finally generate a reduced intracellular $\mathrm{pH}$ able to drive a metabolic switch to OxPhos, an activity mediated by $\mathrm{PI} 3 \mathrm{~K} / \mathrm{Akt} / \mathrm{mTOR}$ signal transduction pathway inhibition of HIF-1 $\alpha$-dependent glycolysis and TCA block $[16,17]$. Thanks to the aerobic glycolysis adopted by proliferating tumor cells and anaerobic glycolysis supported by HIF- $1 \alpha$ stabilization by low $\mathrm{O}_{2}$ tension, oxygen is conserved and possibly used for a "reduced rate" of OxPhos (note that $0.5-1.5 \%$ $\mathrm{O}_{2}$ is the range of maximum expression of HIF- $1 \alpha$, which is also able to support OxPhos) [18]. OxPhos is also considered a major sink for protons, an activity able to control internal $\mathrm{pH}$, quite different from protons generated during ATP hydrolysis produced by glycolysis; these protons are not consumed and generate acidosis. Furthermore, glucose exhaustion caused by glycolytic cells contributes to the metabolic shift of acidic cells toward OxPhos. It is remarkable that low-pH adapted cells when grown in a high glucose medium continue to show higher pyruvate oxidation, indicating that the Crabtree effect is not applicable to acidic cells [19]. Now, to sustain migration, which is a high energy-demanding process, in the absence of a sufficient glucose supply, respiratory cells may use alternative substrates such as lactate, free fatty acids, and amino acids. A metabolic symbiosis among different tumor cell subpopulations, generating and using lactate [20], or tumor cells/stromal cells metabolic cross talk [21, 22], may contribute to tumor cell dissemination. Recently, Dr. VS LeBleu et al. [23] demonstrated that the bioenergetics phenotype of circulating tumor cells endowed with a high migratory ability and EMT profile use OxPhos. We may speculate that when tumor cells reach their target organ, either they may be transiently dormant or, in case of an adequate blood supply, they start to proliferate, reprogramming their metabolic profile back to aerobic glycolysis.

Overall, to target local and disseminated tumor cells, plasticity of metabolic reprogramming stimulated by the tremendous liaison between hypoxia, glucose, and acidosis (Fig. 1) needs to be targeted first, and this is the so-called "metabolic therapy".

Acknowledgements Work is supported by grants from Istituto Toscano Tumori and Ente Cassa di Risparmio di Firenze.

Conflicts of interest None

\section{References}

1. Ward PS, Thompson CB. Metabolic reprogramming: a cancer hallmark even warburg did not anticipate. Cancer Cell. 2012;21:297308.

2. Schulze A, Harris AL. How cancer metabolism is tuned for proliferation and vulnerable to disruption. Nature. 2012;491:364-73.

3. DeBerardinis RJ, Mancuso A, Daikhin E, Nissim I, Yudkoff M, Wehrli S, et al. Beyond aerobic glycolysis: transformed cells can engage in glutamine metabolism that exceeds the requirement for protein and nucleotide synthesis. Proc Natl Acad Sci U S A. 2007;104:19345-50.

4. Weinhouse S. Glycolysis, respiration, and anomalous gene expression in experimental hepatomas: G.H.A. Clowes memorial lecture. Cancer Res. 1972;32:2007-16.

5. Vélez J, Hail Jr N, Konopleva M, Zeng Z, Kojima K, Samudio I, et al. Mitochondrial uncoupling and the reprograming of intermediary metabolism in leukemia cells. Front Oncol. 2013;3:67.

6. Kato Y, Ozawa S, Miyamoto C, Maehata Y, Suzuki A, Maeda T, et al. Acidic extracellular microenvironment and cancer. Cancer Cell Int. 2013;13:89.

7. Coussens LM, Werb Z. Inflammation and cancer. Nature. 2002;420:860-7.

8. Vaupel P, Höckel M, Mayer A. Detection and characterization of tumor hypoxia using $\mathrm{pO}_{2}$ histography. Antioxid Redox Signal. 2007;9:1221-35.

9. Spivak JL. The anaemia of cancer: death by a thousand cuts. Nat Rev Cancer. 2005;5:543-55.

10. Vaupel P, Mayer A. Hypoxia in cancer: significance and impact on clinical outcome. Cancer Metastasis Rev. 2007;26:225-39.

11. Murayama C, Kawaguchi AT, Kamijo A, Naito K, Iwao K, Tsukamoto $\mathrm{H}$, et al. Liposome-encapsulated hemoglobin enhances chemotherapy to suppress metastasis in mice. Artif Organs. 2014;38:656-61.

12. Goda N, Ryan HE, Khadivi B, McNulty W, Rickert RC, Johnson RS. Hypoxia-inducible factor 1alpha is essential for cell cycle arrest during hypoxia. Mol Cell Biol. 2003;23:359-69.

13. Pouysségur J, Dayan F, Mazure NM. Hypoxia signalling in cancer and approaches to enforce tumor regression. Nature. 2006;441: 437-43.

14. Gatenby RA, Gillies RJ. Why do cancers have high aerobic glycolysis? Nat Rev Cancer. 2004;4:891-9.

15. Peppicelli S, Bianchini F, Calorini L. Extracellular acidity, a "reappreciated" trait of tumor environment driving malignancy: perspectives in diagnosis and therapy. Cancer Metastasis Rev. 2014;33:823-32.

16. Mazzio EA, Boukli N, Rivera N, Soliman KF. Pericellular pH homeostasis is a primary function of the Warburg effect: inversion of metabolic systems to control lactate steady state in tumor cells. Cancer Sci. 2012;103:422-32.

17. Kahn BB, Alquier T, Carling D, Hardie DG. AMP-activated protein kinase: ancient energy gauge provides clues to modern understanding of metabolism. Cell Metab. 2005;1:15-25.

18. Denko NC. Hypoxia, HIF1 and glucose metabolism in the solid tumour. Nat Rev Cancer. 2008;8:705-13.

19. Burd R, Wachsberger PR, Biaglow JE, Wahl ML, Lee I, Leeper DB. Absence of Crabtree effect in human melanoma cells adapted to growth at low $\mathrm{pH}$ : reversal by respiratory inhibitors. Cancer Res. 2001;61:5630-5.

20. Semenza GL. Tumor metabolism: cancer cells give and take lactate. J Clin Invest. 2008;118:3835-7.

21. Fiaschi T, Marini A, Giannoni E, Taddei ML, Gandellini P, De Donatis A, et al. Reciprocal metabolic reprogramming through lactate shuttle coordinately influences tumor-stroma interplay. Cancer Res. 2012;72:5130-40. 
22. Pavlides S, Whitaker-Menezes D, Castello-Cros R, Flomenberg N, Witkiewicz AK, Frank PG, et al. The reverse Warburg effect: aerobic glycolysis in cancer associated fibroblasts and the tumor stroma. Cell Cycle. 2009;8:3984-4001.
23. LeBleu VS, O'Connell JT, Gonzalez Herrera KN, Wikman H, Pantel K, Haigis MC, et al. PGC- $1 \alpha$ mediates mitochondrial biogenesis and oxidative phosphorylation in cancer cells to promote metastasis. Nat Cell Biol. 2014;16:992-1003. 